\title{
La política de garantía de ingresos en España
}

\section{Luis Sanzo González}

Departamento de Empleo y Políticas Sociales, Gobierno Vasco

SanzoGLu@euskadi.eus

Zalantzarik gabeko bidegurutzean dago dirusarbideak bermatzeko politika Espainian. Gizartebabeserako sistemaren gabezia sakonak azaleratu ditu 2008. urte osteko finantzen arloko iskanbiletan jatorria izandako enpleguaren krisialdiak, eta oraindik ere ez da zantzurik ageri sistemaren egokitzerako. Artikulu honetan modu orokor batean aurkezten da ongizatearen Estatuaren oinarrizko zutabe honen etorkizunaren inguruko eztabaida egokitzeko komenigarria den testuingurua.

\section{GAKO-HITZAK:}

Pobrezia, langabezia, gizarte-babesa, Diru-sarrerak Bermatzeko Laguntza, erreforma, gastu soziala.
La política de garantía de ingresos en España se encuentra, sin duda, ante una encrucijada. La crisis de empleo posterior a las turbulencias financieras de $\mathbf{2 0 0 8}$ ha mostrado las profundas carencias del sistema de protección existente, sin que por ahora se perfilen con nitidez las vías de actualización de este sistema. Este artículo presenta, de manera genérica, el contexto en el que se conviene situar el debate sobre el futuro de esta parte fundamental de la acción del Estado de bienestar.

\section{Palabras Clave:}

Pobreza, desempleo, protección social, renta de garantía de ingresos, reforma, gasto social. 


\section{Principales características del sistema de garantía de ingresos}

Uno de los aspectos más llamativos del sistema de garantía de ingresos en España es la existencia de dos redes paralelas de prestaciones asistenciales. Se trata, por una parte, de las rentas mínimas autonómicas (RMA) y, por otra, del sistema vinculado a la Administración General del Estado (AGE) que, tanto en el ámbito de la Seguridad Social como de la protección al desempleo, complementa la acción contributiva estatal.

\subsection{Una política inesperada: las rentas mínimas de las comunidades autónomas}

El 6 de marzo de 1989, el Boletín Oficial del País Vasco publicaba el decreto autonómico que regulaba la concesión del llamado ingreso mínimo familiar $(\mathrm{IMF})^{1}$. Era la primera modalidad de renta mínima autonómica que se establecía en España. Como consecuencia de la posterior extensión del modelo al conjunto de las comunidades autónomas españolas (CC.AA.), la iniciativa del Gobierno Vasco iba a tener importantes consecuencias para la política social española. Abría el camino a lo que, en especial durante la reciente crisis económica, ha funcionado como un sistema de última red de garantía de ingresos.

Es importante señalar que las rentas mínimas son una consecuencia inesperada de la atribución de la competencia de asistencia social a las CC.AA. en la Constitución (artículo 148.1.20a) y su asunción, como competencia exclusiva, en los diversos estatutos de autonomía. La introducción de estas prestaciones no respondía, en este sentido, a ningún mandato constitucional expreso ni a una concreta concepción de la protección social prefijada en la Constitución. Las rentas mínimas no pueden considerarse, por tanto, como un elemento consustancial al modelo de protección social previsto en la Constitución, aunque tampoco pueda afirmarse que se opongan a él en ningún sentido. Es simplemente imposible afirmar que sean un resultado consensuado del modelo de desarrollo constitucional. El IMF vasco fue, en realidad, la manifestación de la decisión de una administración autonómica de introducir una política novedosa, aprovechando un marco constitucional y estatutario que no lo impedía, pero que tampoco lo preveía. Y lo que era cierto respecto a la Constitución también lo era respecto al Estatuto: el IMF tampoco respondía a ninguna previsión estatutaria en Euskadi.

De hecho, consciente de la innovación, y siempre temeroso ante el peligro de desbordamiento, el
${ }^{1}$ Decreto $39 / 89$ de 28 de febrero, relativo al Ingreso Mínimo Familiar. Su contenido fue recogido poco tiempo después, y con algunas modificaciones, en la Ley $2 / 1990$, de 3 mayo, de Ingreso Mínimo de Inserción. la iniciativa vasca. La apuesta de los sindicatos UGT y CC.00. por las rentas mínimas, manifestada en su Plataforma Sindical Prioritaria, contribuyó, sin embargo, a la rápida extensión de estas prestaciones, en particular en la dimensión más formal, con la aprobación de leyes y decretos reguladores de las nuevas prestaciones.

Con el tiempo, la Administración General del Estado fue asimilando el desarrollo de estas actuaciones y dio paso a un planteamiento de convivencia de las distintas actuaciones. Pero sería erróneo considerar que haya pretendido integrar el sistema de RMA dentro de su política de garantía de ingresos, buscando un acoplamiento político-jurídico con las prestaciones estatales. En los años y décadas posteriores a la creación del IMF vasco, el Estado seguiría viendo con distancia la actuación de unas comunidades autónomas que, cada una a su manera, emularon la iniciativa vasca.

Esto explica que siga habiendo problemas para articular la relación entre las prestaciones estatales de garantía de ingresos y las RMA. La completa ausencia de contribución financiera directa por parte de la AGE para la financiación de las RMA se une, en este sentido, a la ausencia de intentos de consensuar principios compartidos para delimitar un verdadero sistema conjunto. La manifestación más clara de esta realidad separada es la persistencia del planteamiento de incompatibilidad que presidió, y aún preside, la relación entre los subsidios de desempleo de la AGE - como, en su momento, el Programa Temporal de Protección por Desempleo e Inserción (Prodi) o, en la actualidad, el Programa de Activación para el Empleo (PAE) - y las rentas mínimas. Una realidad que sigue poniendo en duda la idea de que la AGE haya podido considerar en algún momento a estas RMA como parte del sistema general de protección del Estado.

Aunque las rentas mínimas de las CC.AA. tratan en todos los casos de ajustarse al sistema general de prestaciones asistenciales del Estado, resulta, por tanto, discutible que pueda hablarse en España de un sistema mínimamente integrado de garantía de ingresos. Se trata más bien de dos redes paralelas de intervención, más o menos complementarias, con muy escasos elementos de coordinación e integración funcional entre sí.

\subsection{El desarrollo de la acción asistencial del Estado}

La posición del Gobierno de España ante las iniciativas de las CC.AA. se ha caracterizado por una cierta negación -o al menos infravaloración- de la necesidad o utilidad de los programas autonómicos de rentas mínimas, y por la configuración de un sistema asistencial no contributivo propio del Estado, basado en el desarrollo del artículo 41 de la Constitución. Este artículo prevé que el Estado mantendrá un régimen público de Seguridad Social para toda la ciudadanía que "garantice la asistencia y 
prestaciones sociales suficientes ante situaciones de necesidad, especialmente en caso de desempleo".

A lo largo de los años ochenta, y culminando en 1990 con la legislación sobre prestaciones no contributivas, la AGE desarrolló ese modelo asistencial, destinado a proteger a quienes queden fuera del sistema contributivo general, tanto en el ámbito de la atención a la invalidez o la jubilación como, de forma más específica, en el del desempleo. Los tres pilares del modelo, luego adaptado tras la aprobación del sistema de atención a la dependencia, son las siguientes prestaciones: el subsidio asistencial de desempleo, acompañado de la creación del subsidio o renta agraria; el subsidio de garantía de ingresos mínimos de la Ley de Integración Social del Minusválido (LISMI) y las pensiones no contributivas (PNC) por invalidez y jubilación. Estas actuaciones venían a unirse a los complementos de mínimos, a las pensiones contributivas y a las prestaciones por hijo a cargo para consolidar un sistema aparentemente muy amplio de protección, complementario al sistema contributivo general de la Seguridad Social y de protección al desempleo.

En coherencia con la distante percepción de las RMA, durante el periodo de auge económico la persistencia de situaciones problemáticas graves, cercanas a las que contemplaban algunas de esas rentas autonómicas, en especial en el ámbito del desempleo de larga duración, llevó a introducir una nueva prestación: la denominada renta activa de inserción (RAI). Orientada a situaciones de difícil inserción laboral, la RAl se estableció en el Real Decreto $236 / 2000$, que la perfilaba como un programa temporal para la inserción laboral de trabajadores desempleados de larga duración, en situación de necesidad y mayores de 45 años. Tratando de ajustarse a las directrices sobre el empleo de la Unión Europea, el programa trataba de combinar, en una línea cercana a la de las RMA, la garantía de ingresos con medidas adecuadas de inserción laboral.

Con posterioridad, el excepcional incremento del desempleo tras la crisis financiera de 2008 obligaría a diseñar un nuevo tipo de actuaciones: el Prodi, el Programa de Recualificación Profesional (Prepara) y el PAE. Concebidas como actuaciones extraordinarias, el objetivo de estos subsidios asistenciales se vinculó a la necesidad de una intervención especial en un contexto de muy altos niveles de desempleo.

En la actualidad, en la dimensión relacionada con la protección al desempleo el modelo de subsidios asistenciales de la AGE fija, desde una primera lógica de intervención ordinaria, ciertos supuestos en los que se considera conveniente cubrir la ausencia de protección contributiva: fin de la protección en personas con alguna experiencia de cotización; población trabajadora agraria de Extremadura y Andalucía; personas en edades cercanas a la jubilación; población con responsabilidades familiares; y algunos colectivos especiales, tales como la población emigrante retornada, la excarcelada o la afectada por problemas de violencia de género.

En una lógica complementaria, ya sea en relación con colectivos con dificultades estructurales de acceso al empleo (RAl) o en situaciones que se siguen valorando como coyunturales pero que se mantienen a lo largo del tiempo, dada la situación de crisis (PAE en la actualidad), el sistema AGE trata de afrontar supuestos especialmente problemáticos. Éstos se ligan a determinadas circunstancias personales que pueden requerir un apoyo a largo plazo (violencia de género, presencia de discapacidad, ciertos colectivos de mayores de 45 años) o, de forma excepcional, a emergencias asociadas a un desempleo que se prolonga más allá de lo esperado.

\section{El sistema de garantía de ingresos durante la última crisis}

Sin ningún de género de duda, el desarrollo de los acontecimientos posteriores a la crisis financiera ha mostrado los límites del sistema de protección social en España. De forma paradójica, en ese periodo las rentas mínimas autonómicas han sido las únicas que han ganado peso en la atención a las necesidades sociales. Lejos de favorecer un mayor consenso en torno a ellas, aunque fuera con vistas a su reforma y mejora, esta circunstancia ha venido acompañada de una valoración mucho más negativa y pesimista de este sistema de protección de última red.

\subsection{Las rentas mínimas como problema predefinido}

Durante el periodo de crisis, y en muchos ambientes políticos, académicos e incluso sindicales, las rentas mínimas autonómicas se han visto más como un problema que como parte de la solución a los problemas sociales de España. A pesar de que sus críticos insisten en su papel limitado y secundario, las rentas mínimas son percibidas como uno de los aspectos más controvertidos del sistema de protección. Aunque no es el único motivo, esta valoración se asocia en buena medida a las reticencias que inspira su carácter completamente descentralizado, con un desarrollo exclusivo de las RMA por parte de los Gobiernos autonómicos. Desde este punto de vista, las rentas mínimas se configuran y operan de manera diferente según los territorios, dando lugar a sistemas muy diferenciados, no sujetos a ningún proceso o mecanismo de coordinación.

La idea de fragmentación en la protección es el corolario que surge con mayor frecuencia en este tipo de análisis. Se destaca así la heterogeneidad en la capacidad de protección, con diferencias que afectan a la cobertura de las prestaciones, tanto en términos de personas atendidas como de nivel de protección 
garantizado. Esta circunstancia ha creado un nivel de atención muy desigual, con niveles muy reducidos de protección en algunas CC.AA., en general las más afectadas por la pobreza, con menor presupuesto para actuar en este campo. La introducción de las RMA había complicado, además, el de por sí complejo sistema de prestaciones estatales e introducido problemas de equidad en la protección a las personas.

Estas críticas obvian, no obstante, los elementos de racionalización que han permitido delimitar, en la acción de las CC.AA., ciertos elementos de complementariedad entre los sistemas. Entre ellos, cabe señalar la dimensión subsidiaria y complementaria de una actuación que, más que alterar el sistema general, trata simplemente de reducir el impacto de los huecos de protección dejados al descubierto por el sistema general de protección del Estado. El sistema de cuantías de las RMA, por otra parte, ha tendido en gran medida a acoplarse a las establecidas por la AGE en sus subsidios asistenciales. En la misma línea, se observa una voluntad dominante de ajuste a un marco cercano en cuanto a la configuración interna de las RMA, resultado en parte del acoplamiento de las actuaciones de las CC.AA. a las iniciativas más pioneras.

Estos elementos de racionalización han permitido una cierta funcionalidad en el desarrollo de las RMA, con una cierta voluntad de convergencia en aspectos básicos entre las distintas CC.AA., hasta el punto de que parte de las críticas planteadas parecen en ocasiones afectar más al propio concepto de autonomía política. Como ya se ha señalado, la ausencia de mecanismos de coordinación en la gestión de las rentas mínimas no es exclusivamente, ni siquiera principalmente, atribuible a las CC.AA.

Una crítica muy relacionada con la anterior hace referencia a los límites normativos de estas prestaciones sociales, fuertemente condicionadas por las exclusiones que sus normas establecen al acceso de población necesitada a las rentas mínimas. El resultado, salvo algunas excepciones autonómicas, es una capacidad limitada para hacer frente a las necesidades.

En cualquier caso, resulta indiscutible que existen diferencias reales. Éstas se derivan, sobre todo, de la necesidad de ajustar el marco jurídico a las disponibilidades presupuestarias, comparativamente más limitadas en determinadas CC.AA. Pero también inciden elementos más conceptuales, en particular, la diferente definición de la población que se ha de a atender, con CC.AA. con una concepción más universalista de la protección y otras más centradas en la atención a colectivos tradicionalmente excluidos (los llamados 'excluidos sociales').

\subsection{Los límites del sistema de protección estatal como problema real}

La acción de las CC.AA. se caracteriza por la diversidad en su aportación protectora. Sin embargo, la heterogeneidad territorial asociada a las rentas mínimas no es el problema central de la política de garantía de ingresos mínimos en España. La principal razón es que el origen de los problemas que determinan esta heterogeneidad en la cobertura es previo a la existencia de ese sistema, y debe buscarse en los huecos de atención que deja abiertos del sistema general de protección del Estado. En especial en lo relativo a la población desempleada, su principal ámbito de intervención, las RMA existentes se limitan a hacer frente a estos huecos sin cerrar por el sistema general, sin pretensión alguna de interferir en el modo de actuar de la AGE, ni de crear desigualdades que no existieran con anterioridad a su intervención.

El verdadero problema del sistema de garantía de ingresos debe buscarse, por tanto, en el sistema de protección estatal. Aunque el de este ha tenido importantes resultados en la lucha contra la pobreza, en particular entre las personas mayores, no ha sido capaz de responder al aumento de las situaciones de insuficiencia de ingresos que ha provocado el aumento del desempleo. Priorizar la atención al colectivo pensionista se ha traducido, además, en una caída del peso del gasto en prestaciones asistenciales dedicado a la población desempleada (de un $47,4 \%$ en 2010 a un $38,1 \%$ en 2015 ).

En lo relativo a la población desempleada, el sistema estatal destaca por varias carencias. La primera hace referencia a una insuficiente y decreciente cobertura en la atención. Respecto al volumen de población desempleada ${ }^{2}$, el peso de la población parada con acceso a una prestación contributiva se reduce de cifras cercanas al $42-43 \%$ en 2007 y 2008 al $16,6 \%$ en 2015. En la misma línea, entre las personas desempleadas sin prestación contributiva, el nivel de cobertura cae del $60 \%$ de 2007 a cifras cercanas o inferiores al $35 \%$ a partir de 2012 . Aunque el Prodi fue un intento de prevenir en 2010 esta fuerte caída, su mantenimiento se reveló imposible ante la presión europea en favor de las políticas de austeridad.

Una segunda limitación importante se relaciona con la baja cuantía de las prestaciones, con niveles en general situados en torno al $80 \%$ del indicador público de renta de efectos múltiples (Iprem), los hoy casi eternos 426 euros (cercanos a los 429,21 euros de las PNC en media mensual anualizada). Esto plantea notables problemas de acercamiento a los umbrales de pobreza, en especial en las zonas con mayor nivel de los precios (en particular, en grandes urbes expansivas como Madrid o Barcelona).

\footnotetext{
${ }^{2}$ En este artículo, las tasas de desempleo se calculan utilizando el criterio de la Encuesta de Población Activa (EPA) y la Organización Internacional del Trabajo (OIT), no el del paro registrado utilizado por el Servicio de Empleo Público Estatal (Sepe).
} 
El límite señalado es particularmente llamativo en el caso de los hogares con menores, dado que el sistema de prestaciones AGE no prevé complementos por hijos/as o por la presencia de otros adultos dentro del hogar. La baja cuantía de las prestaciones por hijos/as, incluso inferior por término medio a la de 2008 , no permite compensar este déficit esencial en las prestaciones asistenciales por desempleo.

En particular desde mediados de la pasada década, la práctica congelación de estas prestaciones contrasta además con la tendencia expansiva de las prestaciones contributivas en España.

Un tercer límite destacado a la protección frente al desempleo se asocia a los límites temporales en esa protección: la mayoría de las prestaciones asistenciales son de duración limitada en el tiempo.

Un cuarto aspecto relevante es la priorización de la atención, con un sistema que está diseñado para proteger más a algunos colectivos por razones de niveles previos de cotización, edad, responsabilidades familiares o presencia de ciertos acontecimientos vitales (emigración, excarcelación, malos tratos, entre otros). Estos factores condicionan el acceso a las prestaciones. Aunque también lo limitan entre la población extranjera, en este caso el principal elemento diferencial se vincula a la edad: mientras que un $78,6 \%$ de las personas mayores de 45 años desempleadas tienen algún tipo de protección, la proporción es del $39 \%$ entre las de 30 y 44 años y del 16,9\% entre menores de 30 años.

Mucho menos conocidas, las diferencias territoriales en el acceso al sistema de subsidios asistenciales por desempleo son más llamativas de lo que habitualmente se suele considerar. Tomando como referencia el volumen de personas desempleadas sin acceso a prestación contributiva, el sistema asistencial garantiza un acceso a la asistencia cercano o superior al $45 \%$ de la población considerada en Extremadura, Andalucía, Islas Baleares y Navarra. En una posición intermedia, la proporción se sitúa entre el 40,5 y el 42,7\% en La Rioja, Cantabria, Asturias y Galicia; y entre el 37,7 y el 38,4 \%, en Aragón, Castilla y León y la Comunidad Valenciana. El nivel de protección baja a cifras de entre 34,2 y $36,1 \%$ en Canarias y Cataluña; a en torno a 32,5\% en Murcia y País Vasco; y a un mínimo del $\mathbf{2 7 , 9} \%$ en la Comunidad de Madrid. Comunidades como Madrid, Cataluña o el País Vasco se ven afectadas por un nivel de acceso comparativamente reducido a los programas como el RAI o el PAE.

Los datos señalados indican claramente que los problemas de desigualdad territorial en los distintos programas de garantía de ingresos no se limitan a las RMA. El conjunto de límites señalados están en el origen de las dificultades del sistema de protección estatal para hacer frente al aumento de las situaciones de necesidad durante la crisis. Limitado a un gasto cercano al $1 \%$ del PIB, excluidos complementos de mínimos y prestaciones familiares, el sistema asistencial de la AGE tiene un impacto limitado en la prevención de la pobreza.

Esta realidad es la base de la presión que se ha derivado a otras redes de atención, en especial en el ámbito de las rentas mínima, aunque también en las relacionadas con las entidades locales, Cáritas y los bancos de alimentos.

\subsection{La respuesta del sistema autonómico}

Es, por tanto, en el fracaso del sistema asistencial estatal de protección al desempleo en el que es preciso buscar el origen de la mayor intervención de las RMA, en especial tras la supresión del Prodi y la introducción de recortes en el acceso a las prestaciones por desempleo (2010-2011). En un contexto de reducción de la protección estatal a la población desempleada, muchas personas no han tenido otra opción que recurrir a las RMA o a vías alternativas (entidades locales, Cáritas y bancos de alimentos).

A diferencia de lo observado en la crisis de primeros de los años noventa, además, las RMA estaban conceptual y organizativamente más preparadas para, al menos, tratar de hacer frente al reto planteado por la crisis, en especial en las CC.AA. con mayor trayectoria protectora. $Y$ los datos indican que han intentado cumplir su papel. Ahí donde han intervenido, por muy limitada que haya sido esta intervención en ocasiones, la política de RMA ha tenido efectos positivos:

- En primer lugar, las RMA han mejorado el nivel de ingresos garantizados a través de una política de cuantías más adaptada a las necesidades que las de la AGE. A diferencia de las prestaciones asistenciales del Estado, las RMA consideran complementos de cuantías por miembros añadidos de la unidad familiar.

- En segundo lugar, aunque las cuantías para personas solas son en ocasiones muy bajas, apenas algo superiores a los 300 euros en la Comunidad de Madrid o la Comunidad Valenciana, no debe olvidarse que se conceden a personas que no tienen acceso a las prestaciones del sistema estatal. Estas cuantías no han sufrido además el brutal deterioro observado en algunos países. En este sentido, los valores se alejan en todos casos de los 180,99 euros en que se sitúa la prestación equivalente en Portugal.

Las RMA han avanzado además hacia la normalización, con una creciente atención a perfiles de población muy alejados de la imagen tradicional de la exclusión social. En el País Vasco, por ejemplo, un 46,2\% de los hogares con todos sus miembros parados accedían al sistema de rentas mínimas en 2014.

En la dimensión territorial, los datos revelan que en las áreas con mayor impacto de las RMA las tasas 


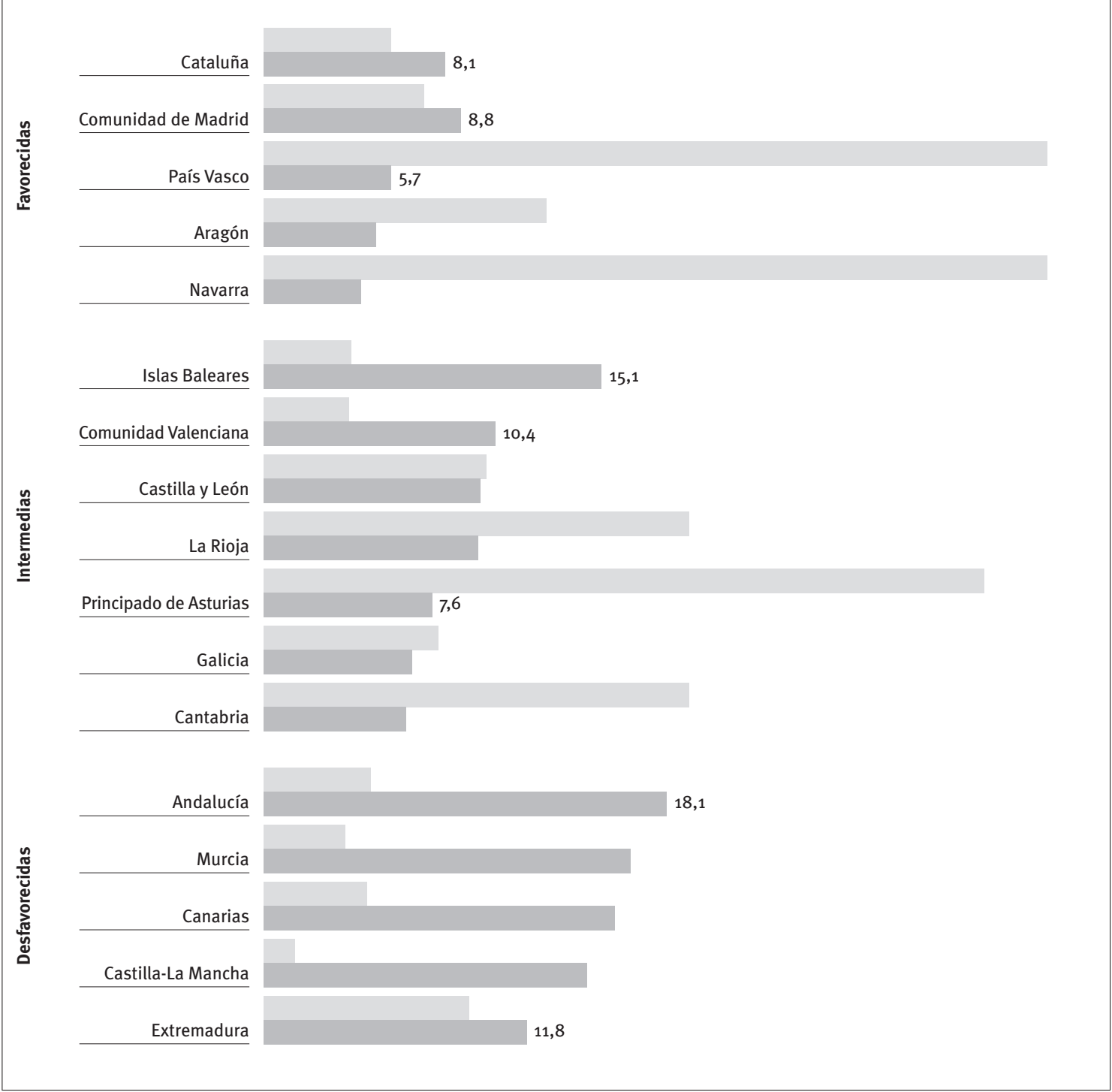

* Impacto de las rentas mínimas autonómicas (\% de cobertura en relación con la población desempleada sin prestación contributiva). ** Tasa de pobreza grave (\% de la población por debajo del 40\% de la renta mediana equivalente en 2015).

Fuente: Elaboración propia a partir de datos del Gobierno de España y el Instituto Nacional de Estadística.

de pobreza resultan más reducidas para cada nivel de posición en la escala socioeconómica territorial (Gráfico 1). Se observa este hecho en las CC.AA. más favorecidas, con tasas de pobreza claramente inferiores en el País Vasco que en la Comunidad de Madrid y Cataluña. La asociación positiva también se observa al comparar, en zonas intermedias, las tasas de las CC.AA. del noroeste español, incluso la de Asturias, respecto a las de la Comunidad Valenciana e incluso Illes Balears. Lo mismo ocurre, en las zonas más favorecidas, al comparar las tasas de pobreza de Extremadura con las de Andalucía, Murcia o Canarias.

Los datos presentados revelan la tendencia a una significativa infraestimación del impacto de las rentas mínimas en España. De hecho, las RMA han dejado de ser el sistema residual que fue durante mucho tiempo. Si se compara la participación de las
RMA en el gasto conjunto que éstas representan, por un lado, y el gasto realizado por los subsidios de desempleo más parecidos, en particular la RAl y los programas extraordinarios (Prodi, Prepara, PAE), por otro, se constata una muy superior importancia cualitativa de estos programas conforme avanzaba la crisis. Así, después de reducirse la participación del gasto en RMA a un 51,3\% en 2009, la introducción del Prodi la situaba en un mínimo del 27,3\% en 2010. Las dificultades de la AGE para mantener el nivel de gasto asociado al Prodi se traducen, sin embargo, en un rápido repunte que, entre 2013 y 2015, lleva la participación de las rentas mínimas a niveles situados entre el $45-47 \%$ del gasto considerado. Como puede comprobarse, en la actualidad el peso del gasto en rentas mínimas es prácticamente similar al que corresponde a los programas especiales de la AGE en materia de desempleo. 


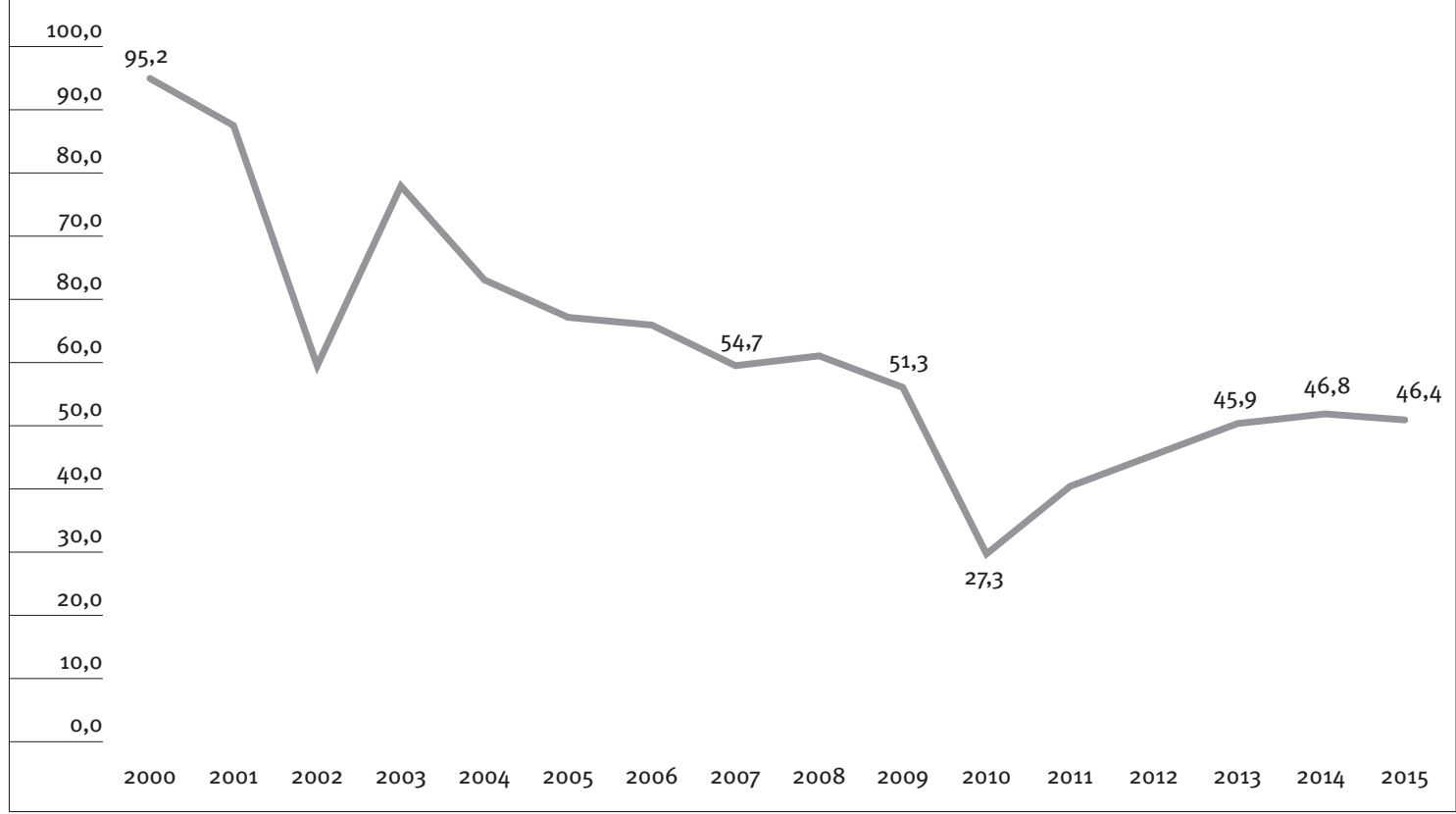

Fuente: Elaboración propia a partir de datos de datos de los Ministerios de Empleo y Seguridad Social y Sanidad, Servicios Sociales e Igualdad.

Los datos relativos a la cobertura de los hogares sin ingresos por las RMA son igualmente significativos. Situada entre $22,7 \%$ y $27,6 \%$ en el periodo 2002 2008 , la proporción avanza del $27,6 \%$ a cifras cercanas al 38,5\% en el periodo 2010-2011. Tras caer al $32,2 \%$ en 2012 , la cobertura repunta a partir de entonces hasta llegar al 43,7\% en 2015.

Aunque los niveles de cobertura son muy diferentes según CC.AA., la atención a las necesidades por parte de las RMA ha sido más importante de lo que habitualmente se admite. A pesar de estas diferencias, no conviene negar la relevancia del sistema de RMA en la atención a la población con dificultades para atender sus necesidades básicas (Sanzo, 2016a):

- Por una parte, son sin duda parte de la explicación a las bajas tasas comparadas de dificultades de acceso a la alimentación que caracterizan a España. Los datos relativos a la proporción de personas en hogares sin acceso a una comida proteínica cada dos días, recogidos para 2013, año de culminación de la crisis, muestran la posición comparativamente favorable de la población española en el Sur de Europa, con una media de $3,5 \%$ que se aleja por completo del $13,8 \%$ de Grecia y del 13,9\% de Italia. También queda por debajo del 7,1\% de Francia.

- Por otra parte, las RMA son un factor que debe tenerse en consideración a la hora de valorar la seguridad económica alcanzada en ciertas zonas. La posición diferencial del País Vasco, Navarra $\mathrm{y}$, en cierta medida, Asturias muestra que estas políticas han tenido un efecto estabilizador a largo plazo en las comunidades que lo han desarrollado. Esto se puede ver con claridad al considerar el indicador de carencia más relacionado con esta seguridad económica a medio y largo plazo, el que refleja la proporción de personas sin recursos suficientes para hacer frente a gastos extraordinarios. El indicador medio de Asturias, Navarra y País Vasco se sitúa en el $25 \%, 11,3$ puntos por debajo de la media de $36,3 \%$ de la zona euro. En la UE, únicamente Suecia se queda dos o más puntos por debajo del indicador medio de las tres CC.AA. señaladas. En fuerte contraste, y a pesar de su potencia económica, la media de Cataluña, la Comunidad de Madrid y la Comunidad Valenciana es 15 puntos superior a la de las tres comunidades del norte mencionadas (40,5\%).

Los datos presentados reflejan, de forma bastante inequívoca, el impacto de una intensidad protectora histórica en las CC.AA. que han apostado por las RMA.

\section{Las alternativas}

La crisis del sistema de protección estatal y la función residual, aunque importante, del sistema de rentas mínimas han llevado a plantear alternativas al modelo de protección social en una forma que no se había observado con anterioridad. Mientras que en las CC.AA. se plantean importantes reformas de las RMA, en el marco español las propuestas se centran en mejoras del sistema de protección estatal. Aunque ningún partido ha llegado a asumir la propuesta, la 
alternativa de la renta básica también se plantea de forma creciente en el debate intelectual y social.

\subsection{La reforma del modelo estatal}

A pesar de los resultados más favorables de lo esperable durante la crisis social, en el contexto español general la reacción dominante no ha sido pedir un incremento de la dotación presupuestaria destinada a las RMA que permitiera consolidarlas como verdaderas prestaciones de derecho en el conjunto del Estado. Se ha impuesto, en cambio, una línea de replanteamiento del sistema general de protección, reforzando la actuación de la AGE en el marco de los preceptos previstos en el artículo 41 de la Constitución. La intención, al menos en parte, sería liberar a unas CC.AA. en cierta forma 'fatigadas' de los compromisos de garantía asumidos en el contexto de sus políticas de rentas mínimas. Este argumento tiene, sin duda, fundamentos objetivos: el aumento de la demanda, en un contexto de fuertes restricciones presupuestarias, ha agotado en buena medida la capacidad de resistencia de las CC.AA. que más se han comprometido con estas políticas.

El objetivo señalado estaba claramente presente en la propuesta del PSOE de creación de una nueva prestación de desempleo, el ingreso mínimo vital (IMV), acompañada de un impulso decidido a las prestaciones familiares que permitiera abordar el reto planteado por la pobreza infantil. En un artículo publicado por El País en febrero del año pasado (Marí-Klose, Moreno Fuentes y Del Pino, 2016), los principales impulsores técnicos de la propuesta señalaban que "las inversiones adicionales que realizaría el Gobierno central en este ámbito liberarían recursos que las comunidades autónomas destinan actualmente a asistir monetariamente a las familias más vulnerables (a través de los programas de rentas mínimas) y que podrían destinar así a favorecer la inserción sociolaboral”. Unos pocos meses antes, en noviembre de 2015, durante el XXII Congreso Anual de la Asociación Estatal de Directoras y Gerentes en Servicios Sociales ${ }^{3}$, la secretaria federal de Políticas Sociales del PSOE había adelantado exactamente la misma idea, aunque mencionando otras posibles líneas alternativas de gasto (vivienda y educación, por ejemplo).

Carlos Bravo, uno de los inspiradores de la iniciativa legislativa popular (ILP) promovida por los sindicatos UGT y CC.00., defendió tesis similares durante el mencionado congreso. Aunque también señaló los límites de la renta activa de inserción, el secretario confederal de Políticas Públicas y Protección Social de CC.00. partió en su análisis, como Carcedo, de una constatación de fracaso de las RMA para garantizar la protección. Se mostró particularmente

$3 \mathrm{El}$ congreso tuvo lugar en Fuenlabrada (Madrid) entre el 6 y el 7 de noviembre. El programa puede consultarse en [<http://www.trabajosocialcordoba.es/archivos/archivos/noticias_general/2152/XXII CONGRESO 2015.pdf>. crítico con la pretensión de las RMA de establecer derechos subjetivos que acaban no siéndolo, al quedar condicionados por las disponibilidades presupuestarias y prácticas dilatorias en la resolución de las solicitudes de las prestaciones. Definió las RMA como derecho aparente que termina, en muchas ocasiones, por no concretarse. Según Bravo, esta realidad ha obligado a reorientar la demanda de soluciones hacia la Administración General del Estado para alcanzar, con carácter estructural, la requerida mejora de la protección.

De hecho, la desconfianza hacia las CC.AA. no puede ser más explícita en la propuesta de prestación contributiva de ingresos mínimos llevada al Congreso por los sindicatos UGT y CC.OO. En la presentación de su modelo, apenas hay dos referencias al papel de esas instituciones en el desarrollo de las políticas de ingresos mínimos. Una de ellas aparece en la exposición de motivos, cuando se dice que deberán contribuir al esfuerzo protector "a fin de que mantengan en algunos supuestos, y refuercen en otros, sus prestaciones de asistencia social". La segunda plantea la posibilidad de que la ejecución de los servicios de la Seguridad Social la desarrollen las CC.AA.

El modelo de gobernanza del IMV partía de una concepción similar, aunque con un planteamiento de pacto para una gestión a través de los servicios de empleo con las CC.AA. En esta aproximación, a las CC.AA. les podría, en todo caso, corresponder una acción de gestión similar a la que, por ejemplo, se desarrolla en materia de prestaciones no contributivas por invalidez o jubilación. Más allá incluso de la propuesta de introducción de una incompatibilidad teórica o práctica entre los sistemas, detrás de todo ello parece encontrarse la oposición a la posibilidad de una actuación complementaria desde las CC.AA. Algo que, de partida, se opone a unos preceptos constitucionales que permiten esta modalidad de intervención autonómica.

Las propuestas de la izquierda tradicional en España, formuladas a través de sus partidos o sus sindicatos, buscan en definitiva consolidar un derecho a la protección desde la Seguridad Social, aunque desde presupuestos similares a los que han inspirado las rentas mínimas. Una parte clave es la introducción de complementos familiares, en la propuesta de UGT y CC.0O., o la mejora de las prestaciones familiares, en la aproximación del IMV.

En cualquier caso, estas propuestas resultan novedosas respecto a las que los principales especialistas españoles en garantía de ingresos planteaban en los momentos iniciales de las crisis. Entonces, investigadores cualificados, como Miguel Laparra y Luis Ayala (2009), planteaban la reforma inmediata de la renta activa de inserción como medida de choque contra la crisis susceptible de afrontar el escenario de desprotección social que se anticipaba. La intensidad de la crisis llevó, sin embargo, a centrar la acción extraordinaria del 
Estado en programas masivos y temporales de protección al desempleo, como el Prodi o el Prepara. El fracaso de estas políticas en la prevención de la pobreza, junto con las dificultades financieras de las CC.AA. en su gestión de las rentas mínimas, ha llevado a reflexiones sobre vías alternativas, en línea con el IMV o la ILP de UGT y CC.OO.

\subsection{La renta básica}

Tal y como se formulaba en 2015 , apenas la propuesta de renta garantizada de Podemos ofrecía por entonces una línea de intervención más posibilista, compatible tanto con el desarrollo de la Seguridad Social como de una participación más amplia de las rentas mínimas autonómicas en el desarrollo de la política estatal de garantía de ingresos mínimos (Álvarez Peralta y Noguera, 2015) ${ }^{4}$. Algo que, interpretado en términos de extensión al conjunto del Estado del modelo de renta mínima de Euskadi, le ganó críticas particularmente duras por parte de los principales ideólogos de la renta básica en España.

En un artículo firmado por Jordi Arcarons, Daniel Raventós y Lluís Torrens (2015) orientado a criticar las opciones asumidas por IU y Podemos y su renuncia a la renta básica, se señalaba que "si algo no tiene la menor duda es la miseria de las rentas para pobres, llámense como se llamen según la comunidad autónoma, como cualquiera que conozca medianamente la cuestión puede constatar". Convertido de repente en paradigma social maligno, los autores señalaban a continuación que la experiencia del sistema vasco dejaba mucho que desear:

Para que no haya la menor confusión sobre lo que queremos afirmar: la CAV ha sido la más generosa de todas las comunidades autónomas en el sistema de rentas condicionadas dirigidas a los pobres. De entrada, esto se debe más a la increíblemente cicatera situación (con alguna excepción parcial, como Navarra) de las otras comunidades autónomas que del mérito de la CAV. Dicho lo cual, está fuera de toda duda que, comparada con la mayor parte de comunidades autónomas, la CAV es la más generosa, pero con múltiples defectos que hemos intentado resumir.

Hablando en nombre de la Red Renta Básica (RRB), en el congreso de noviembre de 2015 ya mencionado, José Luis Rey también asumió la visión crítica, en algún aspecto casi catastrofista, respecto de las rentas mínimas. Las presentó como actuaciones con un balance lamentable, "a lo mejor" con la excepción de las experiencias de Navarra o el País Vasco. Rey realizó una nítida presentación, en cualquier caso, de los argumentos fuertes de la RRB en favor de la

${ }^{4}$ Iván H. Ayala precisó esta propuesta en el XXII Congreso Anual de la Asociación Estatal de Directoras y Gerentes en Servicios Sociales (véase la nota 3). renta básica. Su defensa de esta medida se centró en argumentos relacionados con la necesidad de simplificar el sistema y aumentar su eficiencia. También destacó la conveniencia de superar el papel de "policía" asumido por el trabajo social, evitando los elementos de estigmatización a los que lleva la necesidad de manifestar y demostrar la situación de pobreza. El trabajador social aparece, en el contexto de las rentas mínimas, como un elemento de control, apartado del propio trabajo social. La introducción del principio de la renta básica permitiría, en efecto, evitar la aplicación del principio de control de recursos, uno de los elementos clave de los sistemas de rentas mínimas. Frente a la dimensión paliativa de las RMA, según Rey, la renta básica destacaría además por su carácter esencialmente preventivo.

Otro elemento central en la defensa de la renta básica se vinculó a la individualización frente a la aproximación de hogar de las rentas mínimas tradicionales. En la concepción de la RRB, la renta básica se caracteriza por empoderar a las personas desde una perspectiva individual.

Aunque sostuvo la imposibilidad de postergar la aplicación de la renta básica, José Luis Rey defendió una aplicación progresiva de la medida, abogando como primer paso por una renta básica para menores. Sería una vía para empezar e introducir de forma inmediata mecanismos de renta básica, una propuesta que permitiría enlazar con las defendidas por el PSOE, Podemos o los propios sindicatos.

El planteamiento de prestación universal, individual e incondicional que representa la renta básica se perfila, en cualquier caso, como una alternativa clara a las políticas tradicionales de garantía de ingresos en España. Además de los aspectos positivos señalados por Rey, otra importante aportación de la renta básica se vincula al hecho de que podría facilitar el proceso de acceso de todas las personas necesitadas a las prestaciones, evitando el riesgo del non take up. La renta básica permite, a priori, simplificar los procesos de gestión administrativa asociados en la actualidad a prestaciones como las RMA.

En un contexto de alto endeudamiento, la dificultad de aplicación de la renta básica se vincula, sin embargo, a su coste económico y a la tentación de liquidar el sistema de prestaciones de la Seguridad Social para hacerla viable.

En la propuesta defendida por José Luis Rey, se mantendría el Estado de bienestar, sin sustituir, por tanto, los sistemas de educación, sanidad o dependencia, pero sí se eliminarían las prestaciones situadas por debajo de la cuantía de la renta básica y se tendría en cuenta, a la hora del contraste con el IRPF, el conjunto de los ingresos obtenidos, entre ellos, la aportación íntegra del ahorro, con un tipo fiscal máximo situado en el $49 \%$.

El coste de aplicación fue estimado por José Luis Rey en torno a un $4 \%$ del PIB. En plena fase de boom 
económico, junto a Rafael Pinilla, calculé en 2004 el coste de una renta básica que, gestionada a través del IRPF, mantuviera el actual modelo de prestaciones (Sanzo y Pinilla, 2004). En la aproximación fiscal más razonable, el coste estimado se situó en un 2,66\% del PIB. Dado el fuerte impacto de la crisis, el coste real actual se acercaría, por tanto, a lo señalado por el representante de la RRB.

Sin embargo, la viabilidad política de la renta básica, en la forma planteada por la RRB, no depende solo de su coste, sino de su apuesta por la sustitución del sistema de prestaciones de la Seguridad Social. Se trata de una aproximación que podría resultar muy peligrosa si pudiera llegar a constituir la base de un acercamiento en esta materia entre progresistas y defensores de la liquidación del Estado de Bienestar, al estilo de Charles Murray (Sanzo, 2016b).

\section{Breves consideraciones finales}

En sus 150 Compromisos para mejorar España, el Partido Popular y Ciudadanos ofrecen algunas pistas de por dónde es previsible que se oriente realmente la línea de reforma de la política social en España. En lo relativo a la política de garantía de ingresos, queda claro que resultará básicamente inviable la posibilidad de avanzar, al menos a corto plazo, en medidas como el IMV o la prestación no contributiva de ingresos mínimos planteada por los sindicatos.

Aunque existen muchas dudas sobre qué incremento de cuantías podrían suponer, sí es posible en cambio que mejore la dotación presupuestaria destinada a las prestaciones por hijos/as. Al menos eso es lo que podría hacer pensar la medida 으 63 del acuerdo, que habla de un Plan contra la Pobreza Infantil. Los 5.680 millones previstos para esta actuación, para el conjunto del periodo 2017-2020, no supondrán sin embargo ningún cambio cualitativo determinante: apenas representan algo más de un $10 \%$ de lo que supondría consolidar un sistema digno de rentas mínimas o renta garantizada en España.

En estas circunstancias, es previsible que se mantenga un escenario muy similar al conocido durante la crisis, con una carga muy importante para las dos redes asistenciales que sostienen en España la lucha contra la pobreza: el sistema de prestaciones estatales de la AGE y las rentas mínimas de las CC.AA.

Dos son los retos de futuro que me parece esencial destacar en este contexto. El primero tiene que ver con la reflexión sobre la definición de lo que podría constituir la estructura básica del sistema general de protección y garantía de ingresos. Dentro de ella, cabría sin duda el análisis detallado de las distintas propuestas formuladas, incluyendo no sólo las propuestas de la izquierda tradicional, sino también las distintas propuestas sobre la renta básica.

El segundo reto es más limitado, pero también es más fácil que dé lugar a corto plazo a resultados positivos y favorables para la población. Se trataría, en este caso, de considerar las bases susceptibles de hacer posible un sistema de última red más extendido y eficaz por medio de la reforma de las rentas mínimas autonómicas, ajustándolas a las necesidades de una sociedad moderna de bienestar. Una sociedad que, en este sentido, debería regirse de acuerdo con principios de solidaridad y de garantía de derechos, no sujetos a la aplicación de medidas de contraprestación.

Aunque pequeños y necesariamente residuales en una sociedad con un buen sistema general de Seguridad Social y renta básica, los programas de rentas mínimas seguirán resultando necesarios durante los próximos años. Es inútil perder el tiempo en tratar de desprestigiar un tipo de programas que ha demostrado ser imprescindible para evitar el crecimiento intolerable de las formas más graves de pobreza en nuestro país. 
150 Compromisos para mejorar España [<http://www.pp.es/ sites/default/files/documentos/16.08.28_150_ compromisos_para_mejorar_espana.pdfı].

ÁLVAREZ PERALTA, N.; y NOGUERA, J. (2015) “Un plan contra la desigualdad y la pobreza", El País, 26-10-15 [rhttp://politica. elpais.com/politica/2015/10/25/ actualidad/1445791747_772077.html〉].

ARCARONS, J.; RAVENTÓS, D.; y TORRENS, L. (2015): “El 'trabajo garantizado' de Izquierda Unida y el 'plan de garantía de rentas' de Podemos contra la pobreza: unas propuestas muy pobres", Sin Permiso, 1-11-15 [<http://www.sinpermiso.info/ textos/el-trabajo-garantizado-de-izquierdaunida-y-el-plan-de-garantia-de-rentas-depodemos-contra-la〉].

CONGRESO DE LOS DIPUTADOS (2016) “Proposición de Ley sobre el establecimiento de una prestación de ingresos mínimos en el ámbito de protección de la Seguridad Social (corresponde a los números de expediente 120/000035 de la X Legislatura y 120/000003 de la XI Legislatura)", Boletín Oficial de las Cortes Generales, Congreso de los Diputados, XII Legislatura, Serie B-Proposiciones de ley, 9-10-16, $\mathrm{n}$ 은 3-1 [shttp://www.congreso.es/portal/page/ portal/Congreso/PopUpCGI?CMD=VERLST\& BASE $=$ pu12\&FMT $=$ PUWTXDTS. $f m t \& D O C S=1-$ 1\&DOCORDER $=$ LIFO\&QUERY $=\% 28 B O C G$ 12-B-3-1.CODI.\%29\#(P\%C3\%A1gina1)].

ESPAÑA (2000): “Real Decreto 236/2000, de 18 de febrero, por el que se regula un programa, para el año 2000, de inserción laboral para los trabajadores desempleados de larga duración, en situación de necesidad, mayores de cuarenta y cinco años", Boletín Oficial del Estado, no 6o, 10-3-0o, págs. 9.9169.922 [<https://www.boe.es/buscar/doc. php?id=BOE-A-2000-4608)].

- (1982): “Ley 13/1982, de 7 de abril, de Integración Social de los Minusválidos", Boletín Oficial del Estado, nํㅜ 103, 30-4-82, págs. 11.10611.112 ['https://www.boe.es/buscar/doc. php?id=BOE-A-1982-9983>].
- (1978): “Constitución española”, Boletín Oficial del Estado, no 311, 29-12-1978, págs. 29.31329.424 [/https://www.boe.es/buscar/doc. php?id=BOE-A-1978-31229'].

LAPARRA, M.; y AYALA, L. (2009): El sistema de garantía de ingresos mínimos en España y la respuesta urgente que requiere la crisis social, Madrid, Cáritas; Madrid, Fundación Foessa [<http:// www.foessa.es/publicaciones_download. aspx? $|d=3952\rangle]$.

MARÍ-KLOSE, P.; MORENO FUENTES, F. J.; y DEL PINO, E. (2016): "A grandes males, razonables remedios”, El País, 24-2-16 [khttp:// elpais.com/elpais/2016/02/12/ opinion/1455271013_829169.html/].

PAÍS VASCO (1990): “Ley 2/1990, de 3 de mayo, de Ingreso Mínimo de Inserción”, Boletín Oficial del País Vasco, $\mathrm{n}^{0}$ 106, 30-5-90, págs. 5.107-5.117 [rhttps://www.euskadi.eus/y22-bopv/es/ bopv2/datos/1990/05/9001683a.shtmls].

- (1989): “Decreto 39/89, de 28 de febrero, por el que se regula la concesión del Ingreso Mínimo Familiar", Boletín Oficial del País Vasco, no 44, 6-3-89, págs. 1.362-1.359 [rhttps:// www.euskadi.eus/y22-bopv/es/bopv2/ datos/1989/03/8900574a.shtml)].

SANZO, L (2016a): “El sistema de Rentas Mínimas en España, ¿un verdadero fracaso?”, Hungry Grass, 4-7-16 [<https://hungrygrass. org/2016/07/04/el-sistema-de-rentasminimas-en-espana-un-verdadero-fracaso-luissanzo/s].

- (2016b): “Charles Murray y la Renta Básica”, Hungry Grass, 3-6-16 [rhttps://hungrygrass. org/2016/06/03/charles-murray-y-la-rentabasica-luis-sanzo/>].

SANZO, L.; y PINILLA, R. (2004): La renta básica. Para una reforma del sistema fiscal y de protección social, serie Documentos de Trabajo, no 42/2004, Madrid, Fundación Alternativas [<http://www.fundacionalternativas.org/ public/storage/laboratorio_documentos_ archivos/xmlimport-CMY7Y9.pdf)]. 